\title{
Spin decay and quantum parallelism
}

\author{
John Schliemann, Alexander V. Khaetskii, and Daniel Loss \\ Department of Physics and Astronomy, University of Basel, CH-4056 Basel, Switzerland
}

(Received 24 September 2002; published 4 December 2002)

\begin{abstract}
We study the time evolution of a single spin coupled inhomogeneously to a spin environment. Such a system is realized by a single electron spin bound in a semiconductor nanostructure and interacting with surrounding nuclear spins. We find striking dependencies on the type of initial state of the nuclear spin system. Simple product states show a profoundly different behavior than randomly correlated states whose time evolution provides an illustrative example of quantum parallelism and entanglement in a decoherence phenomenon.
\end{abstract}

DOI: 10.1103/PhysRevB.66.245303 PACS number(s): 76.20.+q, 03.65.Ud, 76.60.Es, 85.35.Be

\section{INTRODUCTION}

The interest in electron spin dynamics in semiconductor structures has increased remarkably in the recent years, generating the emerging field of spintronics. ${ }^{1,2}$ This key word summarizes efforts to use the spins of quantum objects rather than or in combination with their charge for information processing, or, even more ambitious, for quantum information processing. Meanwhile, several proposals for quantum information processing using (electron or nuclear) spins have been put forward. ${ }^{3-7}$

In quantum information processing the coherence of quantum bits is crucial. This issue becomes particularly important in solid state systems where the qubits are ususally assumed to be affected by much more and much stronger perturbating influences than in other experimental setups related to quantum information processing in various other fields of physics such as atomic physics, quantum optics, or NMR experiments. An important advantage solid state systems have, however, is that they offer the possibility of scalability once individual qubits and elementary gate operations between them are estabilshed. Such a perspective is usually not given in other quantum computation scenarios.

Motivated by these developments, in this work we investigate the time evolution of a single spin $\vec{S}$ that is coupled inhomogeneously to a noninteracting environment of other spins. A natural realization of such a system is given by the spin of a single electron in a semiconductor quantum dot interacting with surrounding nuclear spins via hyperfine coupling. ${ }^{8}$ Alternatively one can think of an electron bound to a phosphoros atom implantated into a silicon matrix, ${ }^{5}$ or of other combinations of impurities and host materials such as $\mathrm{Si}$ in a GaAs or Ge matrix. The time evolution of an electron spin under such circumstances is of fundamental interest in its own right, and of particular relevance to the quantum computing proposal of Refs. 3 and 5. In fact, very recently a series of studies of electron spin dynamics related to the present one appeared. ${ }^{9-17}$ Here we build on recent work of Ref. 13, where the dynamics of an electron spin due to the hyperfine interaction with nuclear spins in a semiconductor quantum dot was investigated. This scource of spin decay can be assumed to be the dominant one in a quantum dot geometry where other mechanisms induced by spin-orbit in- teraction are believed to be suppressed, although this issue has not yet been clarified entirely. ${ }^{11}$

Our approach here is based on numerical simulations of the full quantum mechanical spin dynamics in sufficiently small systems. These studies complement the earlier work of Ref. 13, where approximation-free analytical results were obtained for the case of a fully polarized system of nuclei. For a more general initial condition such as an unpolarized nuclear spin system, low-order time-dependent perturbation theory was employed which unfortunately suffers from divergent terms in higher order.

In our numerical simulations we observe a decay of the electron spin as measured in terms of the expectation values of its components. We study this phenomenon as a function of the initial nuclear polarization, and the type of initial state of the nuclear spin system. We compare the time evolutions of initial states, where the nuclear system is in a simple tensor product state, with situations where the initial nuclear state is randomly correlated. A major result is that the time evolution depends very significantly on the type of initial state of the spin environment. The time evolution of simple tensor product states can be quite individual, while randomly correlated (and therefore highly entangled) states show a very reproducible dynamics that mimics the average over the time evolutions of all possible tensor product states. This observation is an example of quantum parallelism in a decoherence phenomenon.

The spin decay is accompanied by the generation of quantum correlations between the electron spin and the nuclear spins, illustrating a general concept of quantum information theory where the decoherece of a quantum bit (here the decay of the electron spin) is viewed as the result of the generation of entanglement (i.e., quantum correlations) between the qubit and its environment. We quantify this entanglement using well-established methods and concepts of quantum information theory. By this we also hope that studies of this kind will faciliate fruitful interactions between the communities of solid state physics and quantum infromation.

Finally, we compare the results of the full quantum mechanical dynamics with simulations of a classical spin model that arises as the classical limit of the underlying Hamiltonian. This comparision shows that the spin decay observed in the quantum system depends crucially on two properties of the system: (i) the inhomogeneity of the hyperfine contact 
interaction induced by the spatial variation of the electron wave function, and (ii) the quantum mechanical nature of the dynamics allowing for nontrivial correlations (entanglement) between the electron spin and the nuclear spins.

This paper is organized as follows: In Sec. II we briefly describe the details of our modeling and technical aspects of our numerical simulations. In Sec. III we report on our numerical results. We discuss the role of different initial conditions for the nuclear spin system, and the connection between decoherence and the generation of entanglement observed in our simulations. We close with conclusions in Sec. IV.

\section{MODEL}

We consider a single spin $\vec{S}$ which is coupled inhomogeneously to a noninteracting environment of other spins:

$$
\mathcal{H}=\vec{S} \cdot \sum_{i} A_{i} \vec{I}_{i}
$$

The coupling is inhomogeneous since the constants $A_{i}$ vary among the environment spins $\vec{I}_{i}$. For an electron spin residing in semiconductor quantum dot the coupling constants $A_{i}$ are given by $A_{i}=A v_{0}\left|\Psi\left(\vec{r}_{i}\right)\right|^{2}$, where $A$ is an overall coupling parameter and $v_{0}$ the inverse density of nuclei in the material. $\Psi\left(\vec{r}_{i}\right)$ is the electron envelope wave function at a location $\vec{r}_{i}$. This factor induces a spatial dependence of the cupliong constants $A_{i}$ which is crucial for the spin dynamics. For simplicity in the following we will consider a nuclear spin of length $I=\frac{1}{2}$ in a spherical quantum dot. In our simulations a given number $N$ of nuclear spins is contained in a sphere of radius $R=\left(3 N / 4 \pi n_{0}\right)^{1 / 3}$, where $n_{0}=1 / v_{0}$ is the density of nuclei. The electron wave function is given by

$$
|\Psi(\vec{r})|^{2}=\left(\frac{1}{\pi(R / a)^{2}}\right)^{3 / 2} e^{-r^{2} /(R / a)^{2}},
$$

where the parameter $a$ describes the confinement of the electron in the dot. In the following we shall use $a=2$ and the material parameters of gallium arsenide with $n_{0}$ $=45.55 \mathrm{~nm}^{-3}$. Therefore, a typical quantum dot contains about $N=10^{5}$ nuclei. For the alternative scenario of an electron bound to a ${ }^{31} \mathrm{P}$ in silicon, the number $N$ of nuclear spins effectively coupled to the electron spin is smaller. The Bohr radius of the hydrogenlike electron state is about $3 \mathrm{~nm}$, and with the lattice constant of silicon and the natural abundance of ${ }^{29} \mathrm{Si}$ this leads to values of $N$ of about a few hundred. To mimic their sperical distribution in systems of smaller size used in our simulations, we also choose the radial coordinate $r_{i}$ of the $i$ th nucleus according to $r_{i}=[3(i$ $\left.-1 / 2) /\left(4 \pi n_{0}\right)\right]^{1 / 3}$, with $i$ ranging from 1 to $N$.

Hamiltonian (1) does not include the direct dipolar interaction between nuclear spins. This interaction is weaker by orders of magnitude than the scale $A$ of the hyperfine coupling, which is of the order $10^{-5} \mathrm{eV}$ in GaAs. ${ }^{8}$ In this material the characteristic time $T_{2 N}$ for the nuclear spin decay due to dipolar interaction is of order $10^{-4} \mathrm{~s}$, while the time scales considered in this work will be at least two orders of magnitude smaller. We also mention a recent interesting numerical study by Dobrovitski et al. ${ }^{18}$ on spin dynamics stressing the role of entropy. There a central spin is coupled inhomogeneously to an essentially non-interacting spin environment, where, differently from the present study, an Isinglike coupling was used. To allow for nontrivial dynamics, the authors of Ref. 18 introduced a magnetic field perpendicular to the $z$ direction of the Ising coupling.

Model (1) was specifically studied recently in Ref. 13, where approximation-free analytical results were obtained for the case of a fully polarized system of nuclei. For a more general initial conditions such as an unpolarized nuclear spin system, low-order time-dependent perturbation theory was employed, which unfortunately suffers from divergent terms in higher order. In this work we choose a different route and perform finite-size exact diagonalizations from which we obtain the full time evolution.

Since our Hamiltonian conserves the total spin $\vec{J}=\vec{S}$ $+\Sigma_{i} \vec{I}_{i}$, it is convenient to work in a subspace of given $J^{z}$ having a dimension of

$$
\left(\begin{array}{c}
N+1 \\
\frac{N+1}{2}-J^{z}
\end{array}\right) .
$$

To obtain the time evolution of the total spin system with the initial state lying in a subspace with a given value $J^{z}$, we diagonalize the Hamitonian within this subspace and compute the time evolution of certain expectation values from the eigensystem data. For initial states having a nonzero overlap in several subspaces with different $J^{z}$ the time evolutions obtained in the different spaces have to be superimposed. The fact that the full eigensystem of the Hamiltonian is required in this procedure is different from most other numerical investigations of spin systems where, for reasons of the physical questions being investigated, it is sufficient to concentrate on the ground state and some low-lying excitations. In our case we need the full eigensystem, and are therefore restricted to system sizes $N$ and values of $J^{z}$ yielding dimensions of not more than a few thousand. However, as we will explain in more detail below, our findings depend neither on the restriction to a certain value of $J^{z}$ nor on the specific coupling constants induced by the quantum dot geometry. In fact, qualitatively the same results are obtained when working in the full Hilbert space, or if the coupling parameters are chosen at random from a uniform distribution. On the other hand, it is essential that the coupling is inhomogeneous, leading to a time evolution which is for all practical time scales aperiodic and in this sense irreversible. The case of homogeneous coupling with all $A_{i}$ being the same is readily solved analytically and generates periodic dynamics with recurrence time $T=4 \pi N \hbar / A$.

\section{RESULTS FOR THE TIME EVOLUTION OF SPINS}

In the time evolutions to be discussed below the initial state will always be a simple direct product of the state of the 


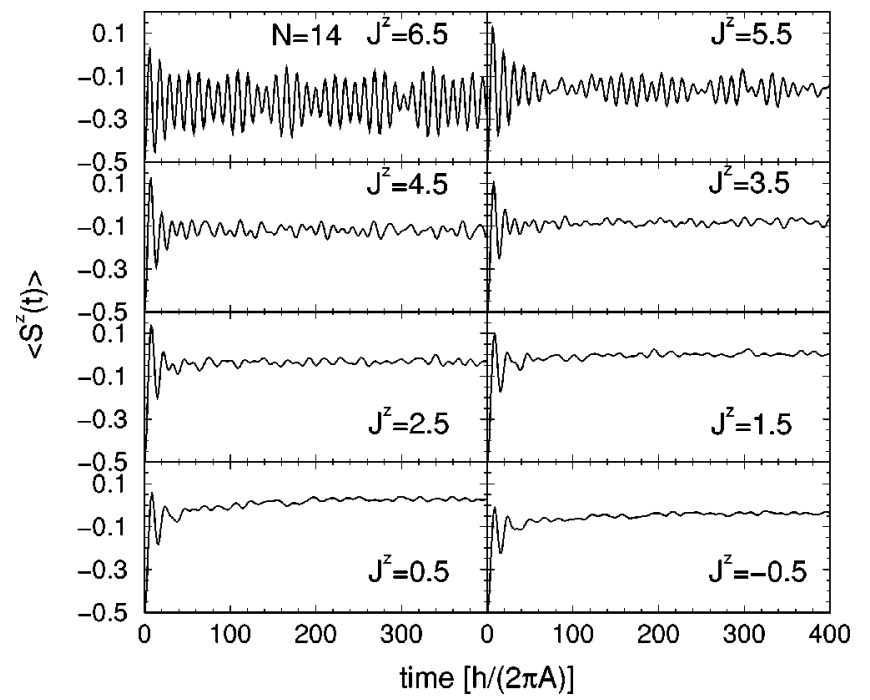

FIG. 1. The time evolution of the electron spin in a system of $N=14$ nuclear spins for different degrees of polarization of the randomly correlated nuclear system and coupling constants induced by the quantum dot geometry. In the top left panel the nuclear spins are fully polarized in the initial state with the electron spin pointing opposite to them $\left(J^{z}=13 / 2\right)$. In the following panels the number of flipped nuclear spins in the initial state is gradually increased. The case of an initially fully unpolarized (but randomly correlated) nuclear system is reached in the bottom right panel $\left(J^{z}=-1 / 2\right)$. Here and in the following we take spins to be dimensionless, i.e. measured in units of $\hbar$.

nuclear or environmental spin system, and the electron spin with the latter pointing downward along the $z$ direction. Therefore, the nuclear spins and the electron spin are initially uncorrelated. For the nuclear spin system itself we consider two types of initial conditions which give rise to significantly different time evolutions.

\section{A. Product states versus randomly correlated states}

We investigate two types of initial states for the nuclear spin system which differ crucially in their corrrelation properties and also, as we shall see below, as a consequence of this in their time evolution: (i) The nuclear spins are initially in a simple tensor product state. If $J^{z}$ is fixed to a certain value such a state consists of eigenstates of $I_{i}^{z}$ for each nuclear spin $i$. If this restriction is not applied tensor product states consisting of more general spin-coherent states are possible. (ii) The nuclear spin state $\left|\chi_{N}\right\rangle$ is initially a linear superposition, $\left|\chi_{N}\right\rangle=\Sigma_{T} \alpha_{T}|T\rangle$, where the sum goes over all tensor product states $|T\rangle$ consisting of eigenstates $I_{i}^{z}, i$ $\in\{1, \ldots, N\}$, and is, for fixed $J^{z}$, restricted to the appropriate subspace. The coefficients $\alpha_{T}$ in this entangled pure state are subject to a normalization condition and chosen either at random or coherently (for example, they can have the same phases).

As we shall see shortly, a single tensor product state, on the one hand, and a randomly correlated nuclear state, on the other hand, generate strikingly different time evolutions for the electron spin. Figure 1 shows numerical data for time-

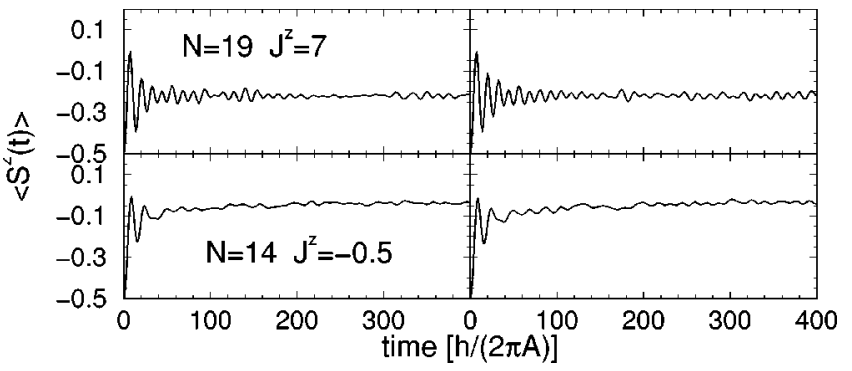

FIG. 2. Upper panels: $\left\langle S^{z}(t)\right\rangle$ for a system of size $N=19$ being initially in a randomly correlated nuclear spin state in the subspace with $J^{z}=7$. The two panels represent two different randomly chosen initial conditions. Lower panels: Analogous data for $N=14$ and a completely unpolarized nuclear spin system $\left(J^{z}=-1 / 2\right)$. In both cases the simulation data does practically not depend on the initial condition.

evolved expectation value $\left\langle S^{z}(t)\right\rangle$ for an initially randomly correlated system and different degrees of its polarization (characterized by $J^{z}$ ). In all cases, $\left\langle S^{z}(t)\right\rangle$ decreases in magnitude. With decreasing polarization the decay becomes more pronounced, and the oscillations accompanying this process are suppressed. Note that it is the decay of the envelope in these graphs but not the fast oscillation itself that signals the decay of the spin. The distance between two neighboring maxima of the oscillations can depend slightly on the initial state and the coupling constants in the Hamiltonian. However, a good estimate of this effective period is usually given by $T=4 \pi \hbar / A$ since $A / 2$ is an estimate (neglecting quantum fluctuations) for the width of the spectrum, i.e., the difference between the largest and smallest eigenvalues of the Hamiltonian.

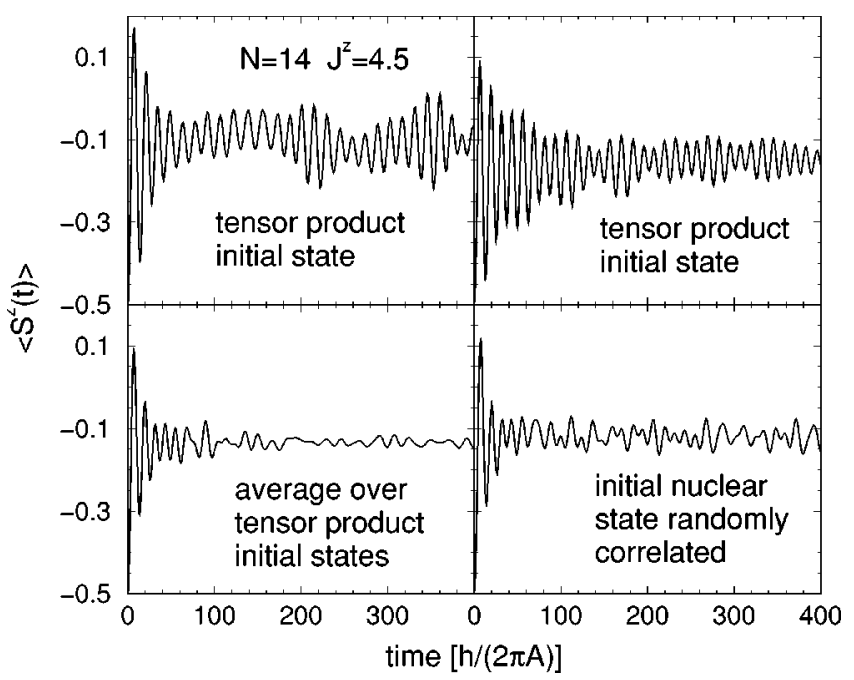

FIG. 3. Upper panels: time evolution of the electron $\operatorname{spin}\left\langle S^{z}(t)\right\rangle$ for a system with 14 nuclear spins being initially in an uncorrelated tensor product state in the subspace $J^{z}=9 / 2$. The oscillation period and the time scale of the decay are consistent with the period $T$ $=4 \pi \hbar / A$ and the scale $\hbar N / A$ identified in Ref. 13. Lower left panel: data of the same type as above but averaged over all possible uncorrelated initial states with $J^{z}=9 / 2$. Here again, the time scale of the decay is consistent with the scale $\hbar \sqrt{N} / A$ identified in Ref. 13. Lower right panel: $\left\langle S^{z}(t)\right\rangle$ for the same system being initially in a randomly chosen correlated state. 
When the nuclear spin system is initially in a randomly correlated state the time evolution of $\left\langle S^{z}(t)\right\rangle$ is very reproducible in the sense that it depends only very weakly on the particular representation of the initial random state. This is illustrated in Fig. 2, where the results of different initial random configurations are compared for two different system sizes and degrees of polarization.

This behavior of randomly correlated initial states sharply contrasts with the time evolution of simple tensor product nuclear spin state. The upper two panels of Fig. 3 show the time evolution of the electron spin for two initial tensor product states. In the lower right panel we compare these data with the time evolution of a representative of the randomly correlated initial condition. In the former case the time evolution depends significantly on the concrete initial tensor product state, and the decay of the electron spin occurs typically clearly more slowly than in the case of an initially randomly correlated nuclear spin system.

In the lower left panel of Fig. 3 we show the time evolution of the electron spin averaged over all nuclear tensor product states. ${ }^{19}$ Comparing the two lower panels one sees that these data are very close to the time evolution of a randomly correlated state. This observation is also made for other system sizes and degrees of polarization, and constitutes an example of quantum parallelism: ${ }^{20}$ The time evolution of each initially uncorrelated (and therefore classicallike) nuclear state is present in the evolution of a linear superposition of all such states. In other words, the time evolutions of all uncorrelated classical-like states are perfomed in parallel in the time evolution of the randomly correlated state. An experimental consequence of this observation is that if the electron spin dynamics would be detected on an array of independent quantum dots, one could not distinguish whether the nuclear spin system in each dot was initially randomly correlated or in an uncorrelated tensor product state. In other words, the spin dynamics of a randomly correlated pure state of the nuclear system in a single dot cannot be distinguished from a mixed state of an ensemble of dots. ${ }^{21}$

The observation that the time evolution of a randomly correlated state quite closely mimics the average over all tensor product initial conditions relies on the cancellation of off-diagonal terms $\alpha_{T}^{*} \alpha_{T^{\prime}}\left\langle\Downarrow, T|\vec{S}(t)| \Downarrow, T^{\prime}\right\rangle, T \neq T^{\prime}$, due to the randomness in the phases of the coefficients $\alpha_{T}$. In this

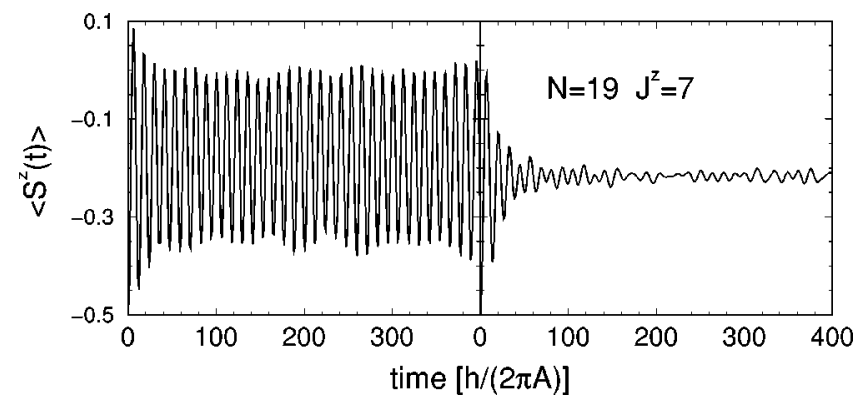

FIG. 4. Time evolution of $\left\langle S^{z}(t)\right\rangle$ for two types of initially randomly correlated nuclear spin states. In the left panel the amplitudes $\alpha_{T}$ are restricted to have non-negative real and imaginary parts, while in the right panel they have all the same modulus but completely random phases. sense our system has a self-averaging property. This can be checked explicitly by reducing this randomness. The left panel of Fig. 4 shows the time evolution of a randomly correlated state where the amplitudes $\alpha_{T}$ are restricted to have non-negative real and imaginary parts. This time evolution turns out to be reproducible similarly to as above i.e., it does not depend on the concrete realization of the initial random state, but is clearly different from the former case since the cancellation of off-diagonal contributions is inhibited. ${ }^{19}$ For comparison, in the right panel we show data in which the amplitudes in the initial nuclear spin state have a random phase but are restricted to have the same modulus. Here the proper averaging process takes place again. The results described so far were obtained in certain subspaces of $J^{z}$ and for the form of coupling constants $A_{i}$ as induced by the quantum dot geometry. However, our findings do not depend on these choices. We have also performed simulations were the initial state has an overlap in the full Hilbert space. For a randomly correlated initial nuclear spin state the only difference is that now transverse components $\left\langle S^{x}(t)\right\rangle$, and $\left\langle S^{y}(t)\right\rangle$ of the electron spin also evolve. However, these are tiny in magnitude and oscillate around zero. For an initial tensor, product states these transverse components can become sizable, and the time evolution again strongly depends on the concrete initial tensor product state. Moreover, as mentioned earlier, the exact form of the coupling constants is also not crucial as long as they are sufficiently inhomogeneous. For instance, we obtain qualitatively the same results if we choose the coupling parameters randomly from a uniform distribution.

We also note that coupling a magnetic field to the electron spin has only a quantitative influence on our results. Here again the time dependence of tensor product initial nuclear state is very individual, while a randomly correlated states gives very reproducible results that mimic closely the average over tensor product states.

\section{B. Decoherence and the generation of entanglement}

In circumstances of quantum information processing the decay of a qubit is usually viewed as some "decoherence" process due to the environment attacking the quantum information. As seen above, the spin decay is generically slower if the spin environment is initially in a uncorrelated state. This finding suggests that it is advantageous for protecting quantum information to disentangle the environment that unavoidably interacts with the qubit system.

A "decoherence" process of the above kind can be viewed as the generation of entanglement between a qubit and its environment. The system investigated here provides an illustrative example of this statement. The entanglement in the total state $|\Psi(t)\rangle$ between the central electron spin and its environment can be measured by the von Neumann entropy of the partial density matrix, where either the electron or the environment has been traced out from the pure-state density matrix $|\Psi(t)\rangle\langle\Psi(t)|{ }^{22}$ Tracing out the nuclear system we have 


$$
\rho_{e l}(t)=\left(\begin{array}{cc}
\frac{1}{2}+\left\langle S^{z}(t)\right\rangle & \left\langle S^{+}(t)\right\rangle \\
\left\langle S^{-}(t)\right\rangle & \frac{1}{2}-\left\langle S^{z}(t)\right\rangle
\end{array}\right) .
$$

This matrix has eigenvalues $\lambda_{ \pm}=1 / 2 \pm|\langle\vec{S}(t)\rangle|$, and the measure of entanglement reads $E[|\Psi(t)\rangle]=-\lambda_{+} \log \lambda_{+}$ $-\lambda_{-} \log \lambda_{-}$. Thus the formation of expectation values $|\langle\vec{S}(t)\rangle| \neq 1 / 2$ [or, in the case of fixed $J^{z}$, just $\left|\left\langle S^{z}(t)\right\rangle\right|$ $\neq 1 / 2]$, is a manifestation of the entanglement between the electron spin and the nuclear spin system. The maximum entanglement, $E=\log 2$, is achieved if the electron spin has decayed completely as measured by the expectation values of its components, $\langle\vec{S}(t)\rangle=0$. The generation of quantum entanglement between the electron spin and the nuclear spin system, signaled by a reduced value of $\langle\vec{S}(t)\rangle$, is the main and crucial difference between the quantum system studied here and its classical "counterpart" described by a system of Landau-Lifshitz equations. These equations can be obtained from the Heisenberg equations of motion for the quantum system, $\partial \vec{S} / \partial t=i[\mathcal{H}, \vec{S}] / \hbar$ and $\partial \vec{I}_{i} / \partial t=i\left[\mathcal{H}, \vec{I}_{i}\right] / \hbar$, by performing expectation values of both sides within spincoherent states and assuming that the expectation values of all operator products factorizes to products of expectation values. This procedure becomes exact in the classical limit. ${ }^{24}$ The resulting equations no longer contain operators, but just describe the dynamics of three-component vectors (classical spins) of fixed length. We have performed simulations of such a classical spin system by solving the Landau-Lifshitz equation via the fourth-order Runge-Kutta scheme. As a result, the central classical spin performs an irregular chaotic motion which does not show any similarity to the results for the quantum spin- $\frac{1}{2}$ case. In particular all qualitative features of quantum effects, such as the generation of entanglement (signaled by a decay of spins as measured by their expectation values), are not present in such a time evolution. Therefore, the Landau-Lifshitz equation provides only a rather poor description of the underlying quantum system.

Let us now briefly discuss how the different initial conditions can be prepared experimentally. A tensor product initial state can be produced by applying a magnetic field and having the underlying crystal lattice at a temperature high enough such that spin-lattice relaxation processes to the nuclear spins are efficient. These interactions with the phonon environment will effectively perform projection-type measurements on each spin, and force the system to be in a state close to a tensor product of nuclear states pointing in each of the two direction along the field axis. Another possibilty is the use of all-optical NMR techniques, as described in Ref. 25. A randomly correlated nuclear state, on the other hand, can be achieved by cooling down the lattice to temperatures where phonon processes are suppressed. Then the highly anisotropic and long-ranged dipolar interaction will produce a sufficiently "disordered" state with a highly irregular pattern of amplitudes when expressed in the tensor product basis, as we have confirmed by explicit simulations of a system of eight nuclear spin placed on the edges of a cube. The highly correlated (or entangled) character of these states can be detected by following the individual nuclear spins in terms of their expectation values $\left|\left\langle\vec{I}_{i}(t)\right\rangle\right|$. This quantity decays from its initial value of $1 / 2$ (in a tensor product initial state), on a time scale determined by the dipolar interaction, ${ }^{23}$ to values typically close to zero. According to the entanglement measure $E$ discussed above, this indicates a strong entanglement between each nuclear spin $\vec{I}_{i}$ and its environment of all other nuclear spins. In both cases the initial state of the full system can be prepared by injecting the electron to the quantum dot from an external lead, or the electron state can be prepared by cooling in a magnetic field and ESR techniques.

We finally consider the nuclear spin correlator $C(t)$ $=\left\langle I^{z}(t) I^{z}(0)\right\rangle, \vec{I}=\Sigma_{i} \vec{I}_{i}$, which can be measured directly by local NMR-like measurements such as magnetic resonance force microscopy. ${ }^{26}$ In a subspace of given $J^{z}$ and the electron spin pointing initially downward this quantity reads $C(t)=\left[J^{z}-\left\langle S^{z}(t)\right\rangle\right]\left(J^{z}+1 / 2\right)$. A realistic initial state will have its dominant weight in a series of subspaces with neighboring $J^{z}$ centered around some value. Then the time evolution of $\left\langle S^{z}(t)\right\rangle$ is very similar in these subspaces, and the dynamics of the total nuclear spin can be mapped out by measuring the electron spin, and vice versa.

\section{CONCLUSIONS}

In summary we have studied the dynamics of a single spin coupled inhomogeneously to a spin environment. As the main result the time evolution depends sensitively on the type of initial state of the spin environment. While the time evolution of simple tensor product states can be quite individual, randomly correlated states show very a reproducible dynamics that mimics the average over the time evolutions of all possible tensor product states. This observation constitutes an example of quantum parallelism in a decoherence phenomenon. This effect is clearly seen for all finite system sizes studied here, and can therefore also be expected to be present in realistic quantum dot systems containing about $N=10^{5}$ nuclei, and also in the thermodynamic limit $N \rightarrow \infty$.

The decay of a single spin in terms of its expectation values is due to the formation of entanglement between this spin and its environment. Since this decay is generally slower if the spin environment is initially in a simple tensor product state (i.e., no entanglement among the environmental spins), our results suggest that it is advantagous for protecting quantum information to disentangle the environment. We expect this result to be of a quite general nature, i.e., it should also be valid for other systems consisting of some central quantum object coupled to a bath of other quantum degrees of freedom.

\section{ACKNOWLEDGMENTS}

This work was supported by the Swiss NSF, NCCR Nanoscience, DARPA, and ARO. 
${ }^{1}$ S.A. Wolf, D.D. Awschalom, R.A. Buhrman, J.M. Daughton, S. von Molnar, M.L. Roukes, A.Y. Chtchelkanova, and D.M. Treger, Science 294, 1488 (2001).

${ }^{2}$ Semiconductor Spintronics and Quantum Computation, edited by D.D. Awschalom, D. Loss, and N. Samarth (Springer, Berlin, 2002).

${ }^{3}$ D. Loss and D.P. DiVincenzo, Phys. Rev. A 57, 120 (1998).

${ }^{4}$ V. Privman, I.D. Vagner, and G. Kventsel, Phys. Lett. A 239, 141 (1998).

${ }^{5}$ B.E. Kane, Nature (London) 393, 133 (1998).

${ }^{6}$ J. Levy, Phys. Rev. A 64, 052306 (2001).

${ }^{7}$ T.D. Ladd, J.R. Goldman, F. Yamaguchi, Y. Yamamoto, E. Abe, and K.M. Itoh, Phys. Rev. Lett. 89, 017901 (2002).

${ }^{8}$ M.I. Dyakonov and V.I. Perel, in Optical Orientation (NorthHolland, Amsterdam, 1984), p. 11.

${ }^{9}$ A.V. Khaetskii and Y.V. Nazarov, Phys. Rev. B 61, 12639 (2000); 64, 125316 (2001)

${ }^{10}$ S.I. Erlingsson, Y.V. Nazarov, and V.I. Falko, Phys. Rev. B 64, 195306 (2001).

${ }^{11}$ D. Mozyrsky, S. Kogan, and G.P. Berman, Phys. Rev. B 65, 245213 (2002).

${ }^{12}$ M. Frasca, Physica E 15, 252 (2002).

${ }^{13}$ A.V. Khaetskii, D. Loss, and L. Glazman, Phys. Rev. Lett. 88, 186802 (2002).

${ }^{14}$ S.I. Erlingsson and Y.V. Nazarov, Phys. Rev. B 66, 155327 (2002).

${ }^{15}$ R. de Sousa and S. Das Sarma, cond-mat/0203101 (unpublished).

${ }^{16}$ I.A. Merkulov, A.I. Efros, and M. Rosen, Phys. Rev. B 65, 205309 (2002).
${ }^{17}$ S. Saykin, D. Mozyrsky, and V. Privman, Nanoletters 2, 651 (2002).

${ }^{18}$ V.V. Dobrovitski, H.A. De Raedt, M.I. Katsnelson, and B.N. Harmon, quant-ph/0112053 (unpublished).

${ }^{19}$ We note that averaging the operator $\vec{S}(t)$ over all tensor product states for the nuclear system with the electron spin pointing downwards is not the same as performing a trace over the appropriate $J^{z}$ subspace since states with the opposite direction of the electron spin are left out.

${ }^{20}$ For an elementary introduction to quantum information theory covering the notion of quantum parallelism see, e.g., A. Steane, Rep. Prog. Phys. 61, 117 (1998).

${ }^{21}$ The fact that the randomly correlated state has a self-averaging property means that, even for a single dot with such a nuclear state, the time evolution of the electron spin can be described by a statistical operator (mixed state) corresponding to an infinite temperature of the nuclear system. We stress, however, that this state is pure and possesses some additional properties (due to its entanglement) which cannot be described by a statistical operator corresponding to some temperature.

${ }^{22}$ C.H. Bennett, H.J. Bernstein, S. Popescu, and B. Schumacher, Phys. Rev. A 53, 2046 (1996).

${ }^{23}$ This time scale is essentially given by the $T_{2 n}$ for a bulk nuclear system.

${ }^{24}$ J. Schliemann and F.G. Mertens, J. Phys.: Condens. Matter 10, 1091 (1998).

${ }^{25}$ G. Salis, D.T. Fuchs, J.M. Kikkawa, D.D. Awschalom, Y. Ohno, and H. Ohno, Phys. Rev. Lett. 86, 2677 (2001).

${ }^{26}$ For a recent overview see A. Suter, D.V. Pelekhov, M.L. Roukes, and P.C. Hammel, J. Mater. Res. 154, 210 (2002) and references therein. 